Wien klin Mag 2021 $\cdot 24: 12-17$ https://doi.org/10.1007/s00740-020-00376-8 Online publiziert: 30 . November 2020 (c) Der/die Autor(en) 2020

\author{
Matthias Kochanek ${ }^{1,2}$. Alexander Shimabukuro-Vornhagen ${ }^{1}$ \\ Dennis A. Eichenauer' ${ }^{1}$ Boris Böll' \\ 'Klinik I für Innere Medizin, Schwerpunkt Internistische Intensivmedizin, Klinikum der Universität Köln, \\ Köln, Deutschland \\ ${ }^{2}$ Klinik I für Innere Medizin, Klinikum der Universität zu Köln, Centrum für Integrierte Onkologie Köln- \\ Bonn, Köln, Deutschland
}

\title{
Kreislauftherapie bei Sepsis - wann, wie und wie viel?
}

Die 2016 publizierte Sepsis-3-Definition beschreibt die Sepsis bzw. den septischen Schock als lebensbedrohliche Organdysfunktion bedingt durch die fehlgesteuerte Immunabwehr des Organismus im Rahmen einer Infektion [21]. Eines der klinischen Symptome einer Sepsis bzw. eines septischen Schocks ist die Kreislaufinstabilität. Dem wurde auch in den neuen Definitionskriterien für den Quick-Sequential-Organ-FailureAssessment(qSOFA)-Score Rechnung getragen. Neben einer eingeschränkten Vigilanz und einer erhöhten Atemfrequenz stellt ein systolischer Blutdruck $\leq 100 \mathrm{~mm} \mathrm{Hg}$ ein wichtiges Kriterium in der Definition dar.

》) Im 1-Stunden-Sepsis-Campaign-Bündel und in der Sepsisleitlinie wird das Kreislaufmanagement besonders betont

Im klinischen Alltag spielt die Kreislaufinstabilität in Rettungsdienst, Notaufnahme, Normalstation und bis hin zur Intensivstation eine große Rolle und steht meist im Fokus des Therapiemanagements. Sowohl in der Erarbeitung der Sepsis-3-Kriterien als auch in unterschiedlichen retrospektiven und prospektiven Studien zeigte sich ein eindeutiger Zusammenhang zwi-

Dieser Beitrag wurde in der Zeitschrift Der Internist 10/2020 • 61:997-1001. https://doi. org/10.1007/s00108-020-00861-6 erstveröffentlicht. Zweitpublikation mit freundlicher Genehmigung der Autoren. schen Blutdruckerniedrigung (sowohl des systolischen als auch des mittleren arteriellen Blutdrucks [MAD]) und der Rate an Organdysfunktionen sowie der sepsisbedingten Mortalität [10, 21, 22]. Folgerichtig wurde im 1-StundenSepsis-Campaign-Bündel sowie in der aktuellen Sepsisleitlinie das Kreislaufmanagement besonders betont $[9,16]$. Der vorliegende Beitrag soll einen kritischen Überblick über die Möglichkeiten der Kreislauftherapie geben, besonders darüber, ab wann und in welchem Maße sie eingesetzt werden sollte.

\section{Richtige Anwendung des Blutdruckgrenzwerts}

Die aktuelle Sepsisleitlinie sieht einen Ziel-MAD von $\geq 65 \mathrm{~mm} \mathrm{Hg}$ vor. Liegt der MAD unter dieser Grenze, sollte eine Kreislauftherapie eingeleitet werden. Die bislang durchgeführten Studien belegen, dass eine Hypotonie unterhalb dieses Werts mit einer erhöhten Mortalität verbunden ist [10]. Diese Grenze darf jedoch nicht absolut gesehen werden, sondern muss individuell der jeweiligen Patientensituation angepasst werden. So konnten Studien zeigen, dass Patienten mit vorbekanntem Hypertonus möglicherweise von höheren Zielblutdruckwerten profitieren [1]. Auch konnte in einer Subgruppenanalyse bei älteren Patienten ( $\geq 75$ Jahre) mit niedrigeren MAD-Werten eine geringere Mortalität aufgezeigt werden [7]. Bislang gibt es keine Studie zu der Frage, wie der kritische Grenzwert des Blutdrucks in der Sep- sis bei chronisch hypotonen Patienten definiert werden sollte.

Für das klinische Management gilt daher, dass bei einem MAD $<65 \mathrm{~mm} \mathrm{Hg}$ und weiteren klinischen Auffälligkeiten im Sinne einer Sepsis eine sofortige adäquate Kreislauftherapie eingeleitet werden sollte.

\section{Optionen der Kreislauftherapie}

Verschiedene Möglichkeiten der Kreislaufstabilisierung stehen zur Verfügung. Sowohl die Sepsisleitlinie als auch die Sepsis-Campaign-Bündel empfehlen folgende Maßnahmen zur Kreislaufstabilisierung.

\section{Volumentherapie}

Die Gabe von Flüssigkeit ist eine der wichtigsten Behandlungsoptionen der Hypotonie im Rahmen des Managements einer Sepsis. Bezüglich der Art der Flüssigkeitssubstitution hat es in den letzten Jahren eine Vielzahl an Publikationen gegeben. Keines der derzeit eingesetzten Volumenersatzmittel wurde formell in Bezug auf Sicherheit und Wirksamkeit evaluiert. Die beiden Hauptgruppen für die Flüssigkeitssubstitution sind Kochsalzlösungen und balancierte kristalloide Flüssigkeiten. Als balancierte kristalloide Lösungen werden viele unterschiedliche Lösungen mit einer Elektrolytzusammensetzung, die der des Plasmas ähnelt, bezeichnet, beispielsweise Ringer-Laktat oder Plasma-Lyte A (Baxter International, 


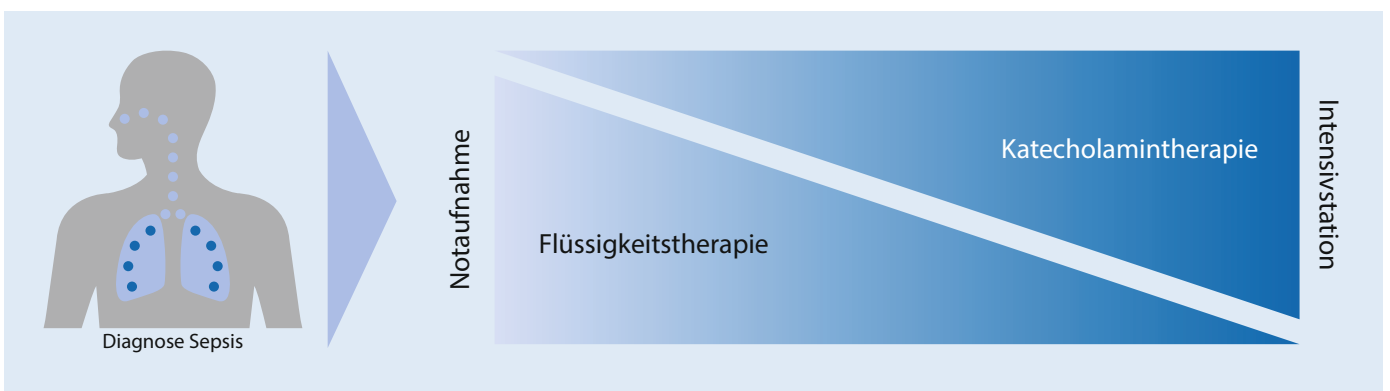

Abb. $1<$ Zusammenspiel des Flüssigkeits- und Katecholaminmanagements zwischen Notaufnahme und Intensivstation
Deerfield, Illinois, USA). Ihnen gemeinsam ist der Mangel an Makromolekülen.

\section{Kristalloide Flüssigkeit}

Die Analyse von Studiendaten liefert unterschiedliche Ergebnisse für verschiedene Patientengruppen. Auf der einen Seite scheint die Art der applizierten Infusionsflüssigkeit die Prognose kritisch kranker Patienten $\mathrm{zu}$ beeinflussen (zu ungunsten der Kochsalzlösung; [14, 19, 20]), auf der anderen Seite gibt es hochrangig publizierte Studien, die keinen signifikanten Unterschied zwischen Kristalloiden und Kochsalzlösungen bei nicht kritisch kranken [18] bzw. kritisch kranken Patienten aufzeigen [24].

\section{》) Kritisch kranke Patienten \\ sollten eher balancierte kristalloide Flüssigkeiten bekommen}

Zusammenfassend kann man sagen, dass bei nicht kritisch kranken Patienten die Art der Flüssigkeitssubstitution keinen großen Einfluss auf das Therapieergebnis hat, wohingegen kritisch kranke Patienten (hier auch Patienten in der Sepsis bzw. im septischen Schock [3]) eher balancierte kristalloide Flüssigkeiten bekommen sollten.

\section{Kolloidale Flüssigkeiten}

Kolloidale Flüssigkeitslösungen, beispielsweise hydroxyethylstärkehaltige oder ähnliche Produkte wie Gelafundin (B. Braun Melsungen, Melsungen, Deutschland), werden explizit nicht zur Volumensubstitution empfohlen. Unterschiedliche Studien konnten zeigen, dass es zu einer eingeschränkten Nierenfunktion bis hin $\mathrm{zu}$ signifikant vermehrten
Nierenersatztherapien und/oder zu einer Mortalitätserhöhung kommt, wenn Kolloide gegeben werden. Die Studienlage für kolloidale Lösungen in der Therapie der Sepsis hat letztendlich dazu geführt, dass neben einer 2013 ausgesprochenen Beschränkung 2018 ein Rote-HandBrief des Bundesinstituts für Arzneimittel und Medizinprodukte (BfArM) verschickt wurde [2].

\section{Albumin}

Obwohl nur mit einer schwachen Evidenz in den Sepsisleitlinien versehen, kann bei großem Bedarf an Flüssigkeit im Rahmen einer Sepsis Albumin eingesetzt werden. Die Saline-versus-Albumin-Fluid-Evaluation(SAFE)-Studie hat gezeigt, dass in einer vordefinierten Subgruppe von Patienten mit schwerer Sepsis die Sterblichkeit bei Gabe von Albumin in der Tendenz niedriger war als bei der alleinigen Gabe von Salzlösung [5]. In der Albumin-Italian-OutcomeSepsis(ALBIOS)-Studie wurde die Wirksamkeit von kristalloider Kochsalzlösung mit und ohne Gabe von $20 \%$ iger Albuminlösung verglichen. Ziel war es, den Albuminspiegel während des Aufenthalts auf der Intensivstation oder bis zu 28 Tage nach Randomisierung auf einem Niveau von $30 \mathrm{~g} / \mathrm{l} \mathrm{zu}$ halten. Es konnten in den primären und sekundären Endpunkten keine signifikanten Unterschiede zwischen den beiden Gruppen festgestellt werden, allerdings zeigte eine Post-hoc-Analyse in der Gruppe der schwerstkranken Patienten mit septischem Schock einen Vorteil zugunsten der Albumingruppe [4]. Eine Studie aus Schweden verglich den Einsatz von $20 \%$ igem und 4 bis $5 \%$ igem Albumin [12]. Als primärer Endpunkt diente zunächst die Menge an applizierter Flüssigkeit innerhalb der ersten $48 \mathrm{~h}$ nach
Randomisierung. Die Ergebnisse zeigten eine Einsparung der innerhalb von $48 \mathrm{~h}$ infundierten Flüssigkeitsmenge. Für den Nachweis eines Überlebensvorteil waren in der Studie keine ausreichend großen Zahlen von Patienten rekrutiert worden.

Viele Metaanalysen und systematische Reviews konnten ebenfalls einen leichten Vorteil für die Therapie mit Albumin herausarbeiten. Allerdings sind all diese Ergebnisse mit Vorsicht zu interpretieren, da unterschiedliche Patientenkollektive (Kinder/Erwachsene), Indikationen, Einschlusskriterien und Krankheitsgrade miteinander verglichen wurden.

Zusammenfassend kann man sagen, dass Albumin in der Standardbehandlung der Kreislaufstabilisierung keine Rolle spielt und nur bei Patienten mit septischem Schock, hohem Flüssigkeitsbedarf und gegebenenfalls niedrigen Albuminspiegeln in Erwägung gezogen werden kann

\section{Katecholamine}

Katecholamine stellen nach wie vor die Therapie der Wahl bei der flüssigkeitsrefraktären Kreislaufinstabilität dar. Eine Vielzahl von Studien und Metaanalysen konnte den Effekt von Noradrenalin auf das Outcome zeigen [16]. Noradrenalin erhöht aufgrund des vasokonstriktiven Effekts den MAD, ohne Herzfrequenz oder Schlagvolumen signifikant zu verändern. Dopamin spielt in der routinemäßigen Behandlung der Kreislaufinstabilität im Rahmen der Sepsis keine oder allenfalls eine marginale Rolle, gegebenenfalls ist es bei Patienten mit einer eingeschränkten Pumpfunktion und einer geringen Wahrscheinlichkeit für Herzrhythmusstörungen indiziert.

Dobutamin kann als positiv inotrope Substanz bei Patienten mit einer einge- 
schränkten Pumpfunktion und gleichzeitig erhaltenem linksventrikulärem Füllungsdruckeingesetzt werden. Allerdings liegen hier auch keine randomisierten Studien im Vergleich zu Placebo vor. Adrenalin zeigt im Vergleich zu Noradrenalin keinen Unterschied im Hinblick auf das Outcome, hat aber eine deutlich höhere Nebenwirkungsrate aufgrund der starken vasokonstriktiven Aktivität und der positiv inotropen Wirkung [15].

\section{》) Vasopressin kann additiv zu Noradrenalin eingesetzt werden}

Vasopressin ist in den letzten Jahren in den Fokus des Managements der Sepsis gelangt. Empfohlen wird die Gabe von maximal 0,03 $\mathrm{U} / \mathrm{min}$, entweder um in Kombination mit Noradrenalin den MAD zu stabilisieren oder um den Bedarf an Noradrenalin zu reduzieren. Bei einer Dosierung oberhalb dieser Grenze ist mit kardialen, digitalen und abdominellen Ischämien zu rechnen [16]. Allerdings liegt für den Einsatz von Vasopressin nur eine geringe Evidenz vor.

Zusammenfassend ist Noradrenalin das Katecholamin der Wahl. Dopamin hat keine und Dobutamin in Ausnahmefällen eine Bedeutung. Vasopressin kann additiv zu Noradrenalin entweder zur Blutdrucksteigerung oder als Mittel zur Reduktion der Noradrenalindosis eingesetzt werden. Hier ist zu beachten, dass die maximale Menge von $0,03 \mathrm{U} / \mathrm{min}$ nicht überschritten werden sollte.

\section{Dosierung der Flüssigkeits- substitution}

Mit Einführung der „early goal-directed therapy“ (EGDT) von Rivers et al. [17] kam es unter anderem zu einem anhand des zentralen Venendrucks (ZVD) gesteuerten Flüssigkeitsmanagement. Dies war über fast 15 Jahre der Goldstandard in der Flüssigkeitssteuerung im Rahmen der Sepsistherapie. Drei große Studien, zwischen 2014 und 2015 publiziert, haben die Elemente dieses Flüssigkeitsmanagements im Rahmen einer Sepsis hinterfragt und kamen zu dem Schluss, dass die protokollbasierte Sepsistherapie mittels EGDT gegenüber der Standardbe-

Wien klin Mag 2021 · 24:12-17 https://doi.org/10.1007/s00740-020-00376-8

(c) Der/die Autor(en) 2020

\section{Kochanek · A. Shimabukuro-Vornhagen · D. A. Eichenauer · B. Böll \\ Kreislauftherapie bei Sepsis - wann, wie und wie viel?}

\section{Zusammenfassung}

Das Management der hämodynamischen Instabilität im Rahmen einer Sepsis bzw. eines septischen Schocks steht in der Notfallversorgung und auf der Intensivstation ganz im Vordergrund. Kreislaufinstabilität hat einen dramatischen Einfluss auf die Rate an Organkomplikationen und die Mortalität bei Sepsis. Nach der Leitlinie zur Therapie der Sepsis soll ein mittlerer arterieller Druck von $65 \mathrm{~mm} \mathrm{Hg}$ nicht unterschritten werden. Kristalloide balancierte Flüssigkeit und
Katecholamine sind die Eckpfeiler des therapeutischen Managements der septischen Kreislaufinstabilität. In diesem Beitrag sollen die wichtigsten Punkte - das Was, Wann und Wieviel - der Kreislauftherapie präsentiert und kritisch diskutiert werden.

\section{Schlüsselwörter}

Septischer Schock · Kreislaufinstabilität . Katecholamine · Flüssigkeitstherapie .

Arterieller Blutdruck

\section{Cardiovascular therapy for sepsis: when, how and how much?}

\section{Abstract}

The management of hemodynamic instability in the context of sepsis or septic shock is at the forefront in emergency care as well as in the intensive care unit. Cardiovascular instability has a dramatic impact on the rate of organ complications and mortality from sepsis. According to the guideline for the treatment of sepsis, mean arterial pressure should not fall below $65 \mathrm{~mm} \mathrm{Hg}$. Crystalloid balanced fluid and catecholamines are the cornerstones of therapy management for septic cardiovascular instability. In this article, the most important points of what, when and how much regarding circulation therapy are presented and critically discussed.

\section{Keywords}

Shock, septic · Cardiovascular system, instability - Catecholamines · Fluid therapy . Blood pressure, arterial handlung durch erfahrene und geschulte Intensivmediziner keinen Überlebensvorteil bringt $[6,13,23]$.

Sowohl in den Sepsis-CampaignBündeln als auch in der Leitlinie werden jetzt initial $30 \mathrm{ml} / \mathrm{kgKG}$ in den ersten 1-3h empfohlen. Anschließend soll in einer Reevaluation der klinischen Situation geklärt werden, ob noch weitere Mengen an Flüssigkeit benötigt werden. An dieser Stelle ist die Leitlinie leider sehr vage gehalten und gibt nur Vorschläge, welche Untersuchungen durchgeführt werden können, um sogenannte „Flüssigkeits-Responder“ $\mathrm{zu}$ detektieren. Um die Volumenreagibilität des Patienten bestmöglich beurteilen zu können, sollte neben der klinischen Beurteilung (Herzfrequenz, Atemfrequenz, Blutdruck etc.) dynamischen Parametern wie der Pulsdruckvariabilität oder Schlagvolumenvarianz bei beatmeten Patienten sowie dem Passive-leg-raising(PLR)-Test der Vorzug gegenüber statischen Parametern (beispielsweise ZVD) oder der gemischtvenösen Sauerstoffsättigung gegeben werden. Ziel ist ein $\mathrm{MAD} \geq 65 \mathrm{~mm} \mathrm{Hg}$. Keines der genannten Verfahren ist allerdings in randomisierten Studien zur Flüssigkeitstherapie im Rahmen einer Sepsis wirklich getestet worden. Eine Studie von Leisman et al. [8] konnte zeigen, dass nur etwa zwei Drittel der Patienten mit Sepsis und Hypotonie volumenreagibel sind, diese aber dann eine um etwa $15 \%$ geringere Mortalität aufweisen als nichtvolumenreagible Patienten. Die Menge von $30 \mathrm{ml} /$ $\mathrm{kgKG}$ ist ebenfalls nicht randomisiert getestet, sondern aus Vorstudien und klinischen Beobachtungen übernommen worden.

Die Balance zwischen zu viel und zu wenig Flüssigkeit ist mitunter schwer $\mathrm{zu}$ finden. Eine überschießende Flüssigkeitsgabe ist ebenso gefährlich wie eine $\mathrm{zu}$ restriktive Gabe und bedeutet eine Schädigung des Patienten. Marik 
Hier steht eine Anzeige.

\section{曾 Springer}


et al. [11] zeigten, dass bei Patienten mit schwerer Sepsis und septischem Schock die Sterblichkeit überproportional zur Krankheitsschwere zunimmt, wenn diese mehr als $6000 \mathrm{ml}$ Flüssigkeit am ersten Tag erhielten. Nachteil dieser sicherlich pointierten Studie ist, dass hier keine Flüssigkeitsbilanz, sondern nur die absolute Flüssigkeitsgabe in der Statistik berechnet wurde ( $\bullet$ Abb. 1).

\section{Fazit für die Praxis}

- Kreislaufinstabilität ist eines der wesentlichen klinischen Symptome der Sepsis bzw. des septischen Schocks. Eine entsprechende Therapie steht deswegen im Vordergrund des Managements der Sepsis.

- Die Sepsis-Campaign-Bündel und die Sepsisleitlinie geben hier bezüglich der Kreislaufstabilisierung folgende Vorgaben:

- Identifikation von Sepsis und septischem Schock

- Sofortiger Beginn der Flüssigkeitstherapie $\mathrm{mit} 30 \mathrm{ml} / \mathrm{kgKG}$ in den ersten 1-3 $\mathrm{h}$ mit Zielwert des mittleren arteriellen Drucks $\geq 65 \mathrm{~mm} \mathrm{Hg}$

- Reevaluation der Volumenreagibilität: cave Flüssigkeitsüberladung!

- Bei unzureichendem Ansprechen auf Flüssigkeitsgabe Beginn einer Vasopressorentherapie (Noradrenalin > Vasopressin)

- Im Hinblick auf die Kreislaufstabilisierung mehren sich die Hinweise, dass ein restriktives Flüssigkeitsregime sowie die frühe Vasopressorentherapie im septischen Schock von Vorteil für die Patienten sind. Dies gilt insbesondere für Patienten, die auf eine Flüssigkeitstherapie nicht mehr ansprechen. Daher ist eine engmaschige Kontrolle der Maßnahmen notwendig, um Schaden vom Patienten abzuwenden.

- Erst der Einsatz aller Therapieoptionen und die Berücksichtigung aller Vorschläge der Sepsisleitlinie bringen den erhofften Erfolg in der Behandlung der Sepsis. Deswegen ist wichtig, dass alle Maßnahmen ineinandergreifen.

\section{Korrespondenzadresse}

PD Dr. Matthias Kochanek

Klinik I für Innere Medizin, Klinikum der Universität zu Köln, Centrum für Integrierte Onkologie Köln-Bonn

Kerpener Str. 62, 50937 Köln, Deutschland matthias.kochanek@uk-koeln.de

Funding. Open Access funding enabled and organized by Projekt DEAL.

\section{Einhaltung ethischer Richtlinien}

Interessenkonflikt. M. Kochanek, A. ShimabukuroVornhagen, D.A. Eichenauer und B. Böll geben an, dass kein Interessenkonflikt besteht.

Für diesen Beitrag wurden von den Autoren keine Studien an Menschen oder Tieren durchgeführt. Für die aufgeführten Studien gelten die jeweils dort angegebenen ethischen Richtlinien.

Open Access Dieser Artikel wird unter der Creative Commons Namensnennung 4.0 International Lizenz veröffentlicht, welche die Nutzung, Vervielfältigung, Bearbeitung, Verbreitung und Wiedergabe in jeglichem Medium und Format erlaubt, sofern Sie den/die ursprünglichen Autor(en) und die Quelle ordnungsgemäß nennen, einen Link zur Creative Commons Lizenz beifügen und angeben, ob Änderungen vorgenommen wurden.

Die in diesem Artikel enthaltenen Bilder und sonstiges Drittmaterial unterliegen ebenfalls der genannten Creative Commons Lizenz, sofern sich aus der Abbildungslegende nichts anderes ergibt. Sofern das betreffende Material nicht unter der genannten Creative Commons Lizenz steht und die betreffende Handlung nicht nach gesetzlichen Vorschriften erlaubt ist, ist für die oben aufgeführten Weiterverwendungen des Materials die Einwilligung des jeweiligen Rechteinhabers einzuholen.

Weitere Details zur Lizenz entnehmen Sie bitte der Lizenzinformation auf http://creativecommons.org/ licenses/by/4.0/deed.de.

\section{Literatur}

1. Asfar P, Meziani F, Hamel J-Fet al (2014) High versus low blood-pressure target in patients with septic shock. N Engl J Med 370:1583-1593

2. https://www.bfarm.de/SharedDocs/Risikoinforma tionen/Pharmakovigilanz/DE/RHB/2018/rhb-hes. html. Zugegriffen:04.02.2020

3. Brown RM, Wang L, Coston TD et al (2019) Balanced crystalloids versus saline in sepsis. A secondary analysis of the SMART clinical trial. Am J Respir Crit Care Med 200:1487-1495

4. Caironi P, Tognoni G, Gattinoni L (2014) Albumin replacement in severe sepsis or septic shock. NEngl JMed 371:84-84

5. Finfer S, Bellomo R, Boyce $\mathrm{N}$ et al (2004) A comparison of albumin and saline for fluid resuscitation in the intensive care unit. $\mathrm{N}$ Engl Med 350:2247-2256
6. Investigators A, Group ACT, Peake SL et al (2014) Goal-directed resuscitation for patients with early septic shock. NEngl J Med 371:1496-1506

7. Lamontagne F, Meade MO, Hebert PC et al (2016) Higher versus lower blood pressure targets for vasopressor therapy in shock: a multicentre pilot randomized controlled trial. Intensive Care Med 42:542-550

8. Leisman DE, Doerfler ME, Schneider SM et al (2018) Predictors, prevalence, and outcomes of early crystalloid responsiveness among initially hypotensive patients with sepsis and septic shock. Crit Care Med 46:189

9. Levy MM, Evans LE, Rhodes A (2018) The surviving sepsis campaign bundle: 2018 update. Crit Care Med 46:997-1000

10. Maheshwari K, Nathanson BH, Munson SH et al (2018) The relationship between ICU hypotension and in-hospital mortality and morbidity in septic patients. Intensive Care Med 44:857-867

11. Marik PE, Linde-Zwirble WT, Bittner EA et al (2017) Fluid administration in severe sepsis and septic shock, patterns and outcomes: an analysis of a large national database. Intensive Care Med. https://doi.org/10.1007/s00134-016-4675-y

12. Martensson J, Bihari S, Bannard-Smith J et al (2018) Small volume resuscitation with $20 \%$ albumin in intensive care: physiological effects : the SWIPE randomised clinical trial. Intensive Care Med 44:1797-1806

13. Mouncey PR, Osborn TM, Power GS et al (2015) Trial of early, goal-directed resuscitation for septic shock. N Engl J Med 372:1301-1311

14. Myburgh J (2018) Patient-centered outcomes and resuscitation fluids. N Engl J Med 378:862-863

15. Myburgh JA, Higgins A, Jovanovska A et al (2008) A comparison of epinephrine and norepinephrine in critically ill patients. Intensive Care Med 34:2226-2234

16. Rhodes A, Evans LE, Alhazzani W et al (2017) Surviving sepsis campaign: international guidelines for management of sepsis and septic shock: 2016. Crit Care Med 45:486-552

17. Rivers E, Nguyen B, Havstad Setal (2001) Early goaldirected therapy in the treatment of severe sepsis and septic shock. N Engl J Med 345:1368-1377

18. Self WH, Semler MW, Wanderer JP et al (2018) Balanced crystalloids versus saline in noncritically ill adults. NEngl J Med 378:819-828

19. Semler MW, Kellum JA (2019) Balanced crystalloid solutions. Am J Respir Crit Care Med 199:952-960

20. Semler MW, Self WH, Wanderer JP et al (2018) Balanced crystalloids versus saline in critically ill adults. N Engl J Med 378:829-839

21. Singer M (2016) The new sepsis consensus definitions (Sepsis-3): the good, the not-so-bad, and the actually-quite-pretty. Intensive Care Med 42:2027-2029

22. Vincent J-L, Nielsen ND, Shapiro NI et al (2018) Mean arterial pressure and mortality in patients with distributive shock: a retrospective analysis of the MIMIC-III database. Ann Intensive Care 8:107

23. Yealy DM, Kellum JA, Huang DT et al (2014) A randomized trial of protocol-based care for early septic shock. NEngl J Med 370:1683-1693

24. Zayed YZM, Aburahma AMY, Barbarawi MO et al (2018) Balanced crystalloids versus isotonic saline in critically ill patients: systematic review and meta-analysis. jintensive care 6:51 
Hier steht eine Anzeige.

\section{曾 Springer}

